\title{
Particularities of changes in internal structure of nanocrystalline Ni under mechanical loading
}

\author{
Dmitrij Kryzhevich ${ }^{1, *}$, Aleksandr Korchuganov ${ }^{1}$, and Konstantin Zolnikov ${ }^{1}$ \\ ${ }^{1}$ Institute of Strength Physics and Materials Science SB RAS, 634055 Tomsk, Russia
}

\begin{abstract}
Molecular dynamics study of the plasticity nucleation mechanisms in a $\mathrm{Ni}$ nanocrystalline sample under shear loading in the constrained conditions was carried out. The studied Ni sample consisted of nine grains of the same size with large misorientation angles relative to each other. In one of the directions, grippers were simulated, to which compressive forces and shear with a constant velocity were applied. In two other directions, periodic boundary conditions were used. It is shown that plasticity nucleation occurs in the region of the triple junction. At the same time, in the region of the triple junction, in the zone of which the stacking fault will be formed, tensile stresses are realized along one of the adjacent grain boundaries, and compressive stresses occur along the other. An increase in stresses in the triple junction zone leads to the formation of a stacking fault, which moves to the volume of one of the grains. Another mechanism of plasticity in nanocrystalline nickel is the migration of grain boundaries, which leads to a significant change in grain sizes.
\end{abstract}

\section{Introduction}

Increased attention to nanocrystalline and nanoscale materials is due to their high strength and functional properties and the possibility of miniaturization of devices of various kinds in electronics, medicine, robotics, etc. Nanocrystalline metallic materials already have wide practical application. At low temperatures, they are characterized by high strength due to grain-boundary hardening (the Hall-Petch effect). At high temperatures, nanocrystalline materials have superplasticity due to grain-boundary softening, which greatly facilitates technological processes. Experimental and theoretical studies [1-6] indicate a high demand for fundamental studies of the mechanisms of plasticity in nanocrystalline materials.

Due to the rather large Peierls barrier, dislocation glide in nanocrystalline metals is significantly suppressed [7-10]. At the same time, the role of various modes of grain boundary-mediated deformation or twinning is enhanced [11-14]. Intergranular sliding becomes the key mechanism of grain boundary-mediated deformation, which prevails during superplastic deformation. Computer simulation [15] and experimental studies [7] indicate a significant contribution of intergranular sliding to the plasticity of nanocrystalline materials at high strain rates and high stress values.

Grain boundary sliding is realized by means of plastic shears along the grain boundaries and leads to the formation of various defects in the region of triple junctions of grain

\footnotetext{
* Corresponding author: kryzhev@ispms.ru
} 
boundaries. These defects are sources of internal stresses [16], which, in turn, can lead to the initiation of cracks and brittle fracture.

Simultaneously with the grain-boundary slip, accommodative processes develop in nanocrystalline materials, which transform the nucleated defects and reduce the stress in their regions, thereby increasing the ductility of the material. Therefore, much attention is paid to the study of the nature of accommodation processes in nanocrystalline materials. The emission of lattice dislocations from the zone of triple junctions, diffusion, fragmentation, rotational deformation, splitting, and migration of grain boundaries are typical examples of accommodative mechanisms [17,18]. Migration and splitting of grain boundaries, initiated by grain boundary sliding, are often implemented as a collective mechanism and significantly increase the efficiency of the accommodative process.

We note that most of the work on the study of the mechanical properties and plasticity mechanisms of nanocrystalline materials is based on the use of computer simulation [1924]. A number of issues of the deformation behavior of nanocrystalline metals and alloys are still controversial. This is largely due to the large variety of composition and internal structure of such materials and the difficulties of their experimental study at the microscopic level. In this connection, in the present work a molecular dynamics study of the mechanisms of nucleation and development of plasticity in nanocrystalline $\mathrm{Ni}$ under conditions of high-speed deformation was carried out.

\section{Methods and setup}

The task was solved within the framework of the molecular dynamics approach using the LAMMPS software package [25]. The calculations were performed for a Ni nanocrystalline sample consisting of 9 grains of approximately the same size with large misorientation angles relative to each other. In one of the directions of the sample rigid grips were simulated to which compressive forces and constant speed shear were applied. Periodic boundary conditions were used in two other directions. The simulated sample had the shape of parallelepiped and contained about 1 million atoms (Fig. 1). The interatomic interaction was described with a many-body potential calculated in the framework of the embedded atom method [26]. The initial sample temperature was 300K. Hard grippers shifted with a speed $V$ of $5 \mathrm{~m} / \mathrm{s}$ in opposite directions. The pressing force $F$ acting on each gripper was $0.01 \mathrm{eV} / \AA$. The visualization of the calculation results was performed by the graphic package OVITO [27]. The structure analysis was carried out using the common neighbor analysis [28].

\section{Simulation results}

The simulation results show that the nucleation of plasticity in a Ni nanocrystalline sample begins with the formation of a stacking fault in the region of one of the triple junctions. At the same time, one of the grain boundaries, adjacent to the triple junction, experiences tensile stress, and the other experiences compressive one (Fig. 2). Such a redistribution of stresses favors the nucleation of stacking fault, on opposite sides of which internal stresses of different signs are always realized. During the loading, the intrinsic stacking fault grows into the body of one of the grains (Fig. 2). The position of partial dislocation creating the stacking fault in the grain at various points in time is also clearly seen from the local distribution of $\sigma_{Y Y}$ stresses inside the plastically deformed grain in the vicinity of the stacking fault, which is indicated by a dotted circle in Fig. 3. 


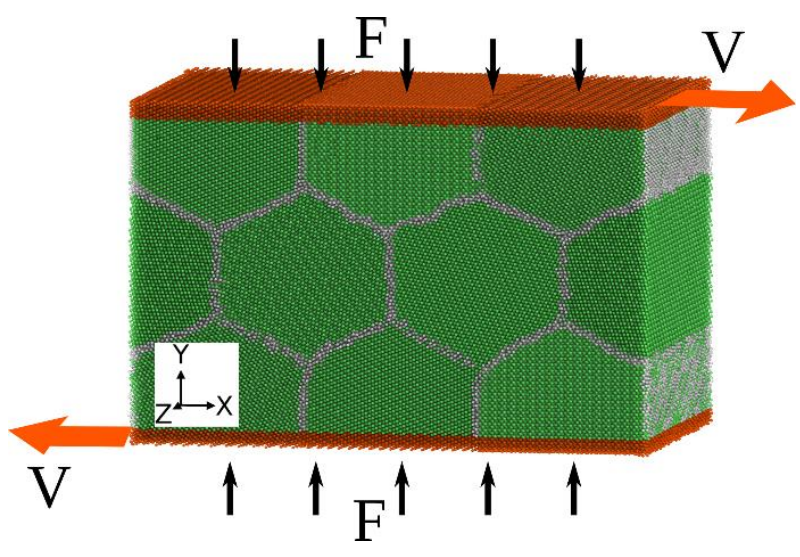

Fig. 1. The initial structure of the sample and the loading scheme. $F$ is the pressing force, $V$ is the shear velocity of grippers.

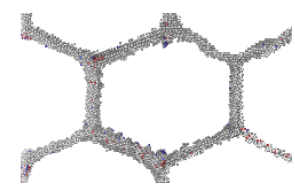

a)

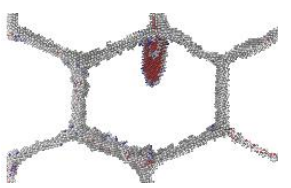

b)

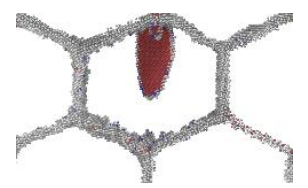

c)

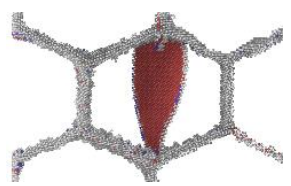

d)

Fig. 2. Fragment of the structure with the stacking fault (highlighted in red) at different points in time: a) 75 , b) 85 , c) 86 , d) 87 ps. The atoms belonging to the grains are not shown.
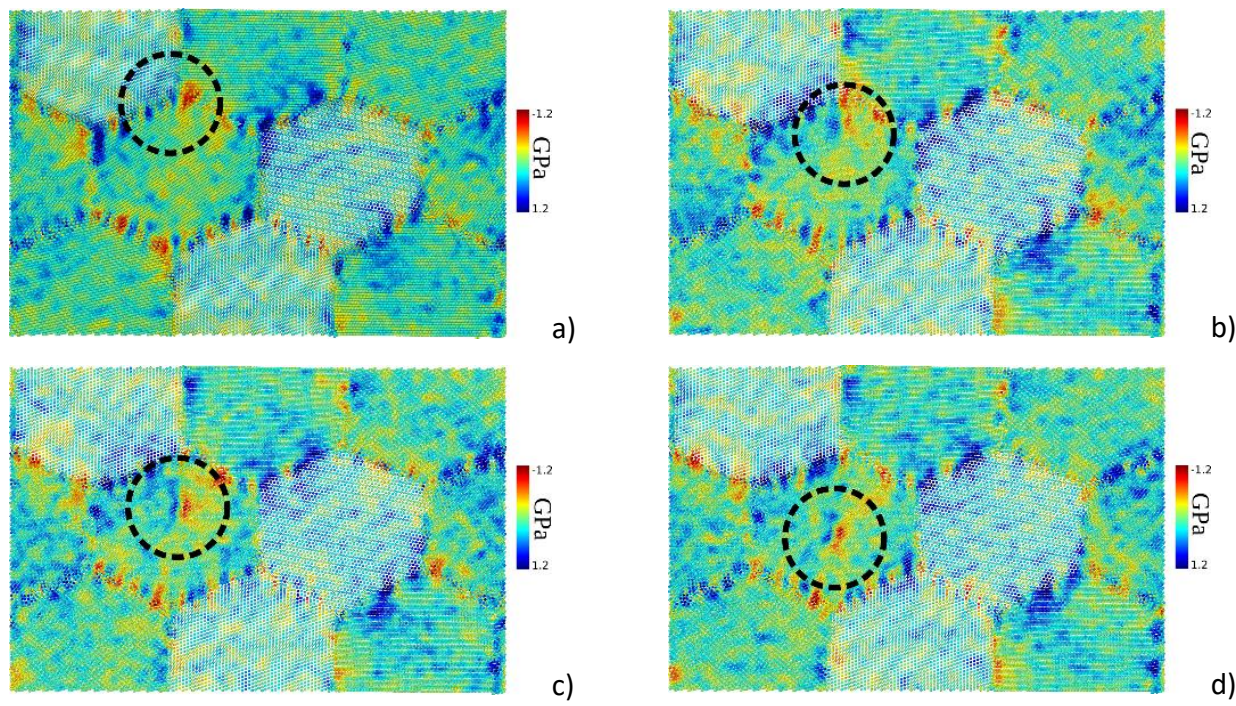

Fig. 3. $Y Y$ stress distribution at different time points: a) 75 , b) 85 , c) 86 , d) 87 ps. The dotted circle shows the area with partial dislocation creating stacking fault.

Note that simultaneously with the generation of stacking faults in the sample, there is an increase in the number of atoms with hcp and an indefinite type of structure of the nearest environment (Fig. 4a). The first kinks on the curves of the number of such atoms (Fig. 4a) 
correlate well with the kinks on the curve of the potential energy change with time (Fig. 4b). This agrees well with the results of other modelling experiments [29]. It should be noted that the origin of stacking fault is the first of the realized mechanisms of sample accommodation for loading. When it is exhausted, the accommodative mechanism connected with the migration of grain boundaries is involved. In Fig. 5 it is clearly seen that during loading the grain boundaries migrate which leads to a significant redistribution of grain sizes.
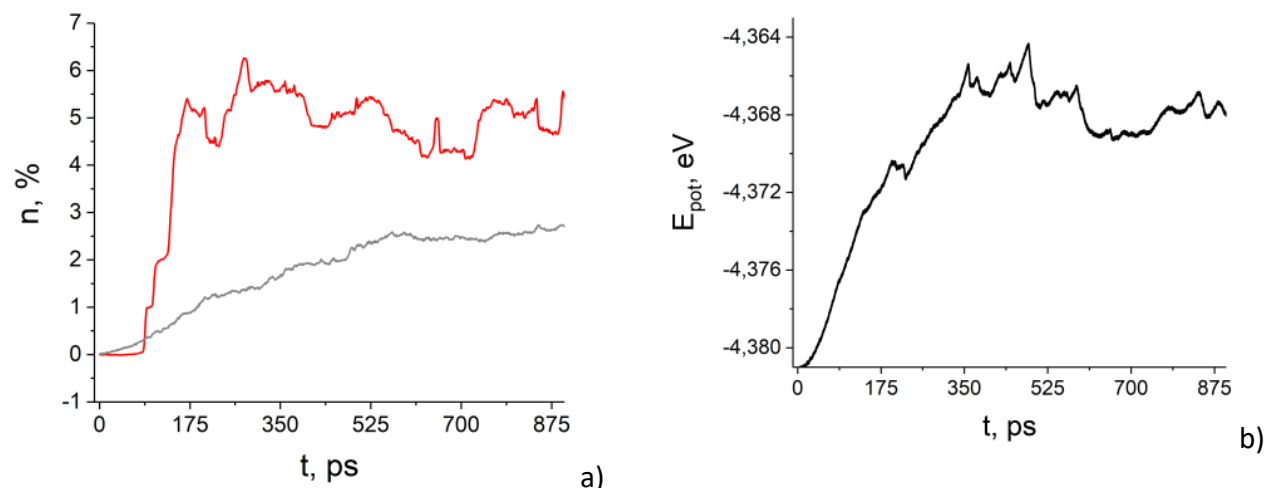

Fig. 4. Fraction of atoms with a hep (red curve) and indefinite (gray curve) symmetry of the nearest environment versus time (a). Potential energy per atom versus time (b).
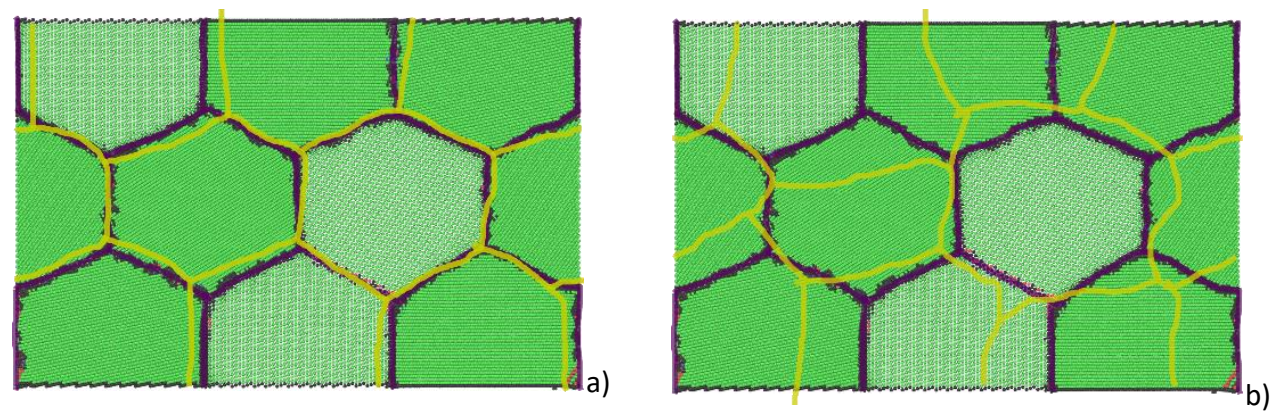

Fig. 5. Grain structure at time points: a) 175 and b) 700 ps. The initial position of the grain boundaries is shown in black, current one in yellow.

\section{Conclusions}

The results of molecular dynamics simulation showed that the main accommodative mechanisms of nanocrystalline $\mathrm{Ni}$ with the same grain sizes and large misorientation angles are the formation of structural defects in triple grain junctions and the migration of grain boundaries. It was found that the formation of stacking faults is preceded by the occurrence of stresses of different signs along the adjacent grain boundaries in the zone of the triple junction. The potential energy versus time curve for a deformed sample has numerous kinks that are associated with accommodative processes. After the exhaustion of the accommodation resource associated with the generation of defects in the triple junction, the processes of migration of grain boundaries are involved. As a result, in the sample there is a significant increase in the size of some grains at the expense of the volume of other grains. 
Studies were performed under Fundamental Research Program of the State Academies of Sciences for 2013-2020 (line of research III.23).

\section{References}

1. M.A. Meyers, A. Mishra, D.J. Benson, Prog. Mater. Sci. 51, 427 (2006)

2. G.A. Malygin, Phys. Solid State 49, 1013 (2007)

3. A.I. Dmitriev, A.Y. Nikonov, A.E. Filippov, V.L. Popov, Phys. Mesomech. 21(5), 419 (2018)

4. M. Dao, L. Lu, R.J. Asaro, J.T.M. De Hosson, E. Ma, Acta Mater. 55, 4041 (2007)

5. S.G. Psakhie, E.V. Shilko, S.V. Astafurov, Tech. Phys. Lett. 30, 237 (2004)

6. I.Yu. Litovchenko, A.N. Tyumentsev, M.I. Zahozheva, A.V. Korznikov, Rev. Adv. Mater. Sci. 31, 47 (2012)

7. A.Y. Nikonov, A.M. Zharmukhambetova, A.V. Ponomareva, A.I. Dmitriev, Phys. Mesomech. 21(1), 43 (2018)

8. I.A. Ovid'ko, J. Mater. Sci. 42, 1694 (2007)

9. D. Wolf, V. Yamakov, S.R. Phillpot, A.K. Mukherjee, H. Gleiter, Acta Mater. 53, 1 (2005)

10. C.C. Koch, J. Mater. Sci. 42, 1403 (2007)

11. X.L. Wu, Y.T. Zhu, Phys. Rev. Lett. 10a1, 025503 (2008)

12. Y.T. Zhu, X.L. Wu, X.Z. Liao, J. Narayan, S.N. Mathaudhu, L.J. Kecskes, Appl. Phys. Lett. 95, 031909 (2009)

13. K.P. Zolnikov, A.V. Korchuganov, D.S. Kryzhevich, Comput. Mater. Sci. 155, 312 (2018)

14. A.V. Korchuganov, A.N. Tyumentsev, K.P. Zolnikov, I.Yu. Litovchenko, D.S. Kryzhevich, E. Gutmanas, S. Li, Z. Wang, S.G. Psakhie, J. Mater. Sci. Technol. 35, 201 (2019)

15. J. Monk, B. Hyde, D. Farkas, J. Mater. Sci. 41, 7741 (2006)

16. N.F. Morozov, I.A. Ovid'ko, Yu.V. Petrov, A.G. Sheinerman, Rev. Adv. Mater. Sci. 19, 63 (2009)

17. N.V. Chertova, Y.V. Grinyaev, Phys Mesomech 21(3), 208 (2018)

18. A.I. Dmitriev, A.Yu. Nikonov, A.R. Shugurov, A.V. Panin, Appl. Surf. Sci. 471, 318 (2019)

19. S.G. Psakhie, K.P. Zolnikov, D.S. Kryzhevich, A.V. Korchuganov, Sci. Rep. 9, 3867 (2019)

20. D.S. Kryzhevich, K.P. Zolnikov, A.V. Korchuganov, Comput. Mater. Sci. 153, 445 (2018)

21. A.I. Dmitriev, A.Yu. Smolin, V.L. Popov, S.G. Psakhie, Phys. Mesomech. 12(1-2), 11 (2009)

22. K.P. Zolnikov, A.V. Korchuganov, D.S. Kryzhevich, S.G. Psakhie, Phys. Mesomech. 21(6), 492 (2018)

23. A. Shugurov, A. Panin, A. Dmitriev, A. Nikonov, Wear 408-409, 214 (2018)

24. K.P. Zolnikov, D.S. Kryzhevich, A.V. Korchuganov, Lett. Mater. 9(2), 197 (2019)

25. S. Plimpton, J. Comput. Phys. 117, 1 (1995)

26. Y. Mishin, D. Farkas, M.J. Mehl, D.A. Papaconstantopoulos, Phys. Rev. B 59, 3393 (1999)

27. A. Stukowski, Model. Simul. Mater. Sci. Eng., 18, 015012 (2010)

28. J.D. Honeycutt, H.C. Andersen, J. Phys. Chem. 91, 4950 (1987)

29. A.V. Korchuganov, K.P. Zolnikov, D.S. Kryzhevich, Mater. Lett. 252, 194 (2019) 